\title{
Correction to: Intra-ligamentary autologous conditioned plasma and healing response to treat partial $\mathrm{ACL}$ ruptures
}

\author{
Matthias Koch ${ }^{1} \cdot$ Berardo Di Matteo ${ }^{3}$. Jürgen Eichhorn ${ }^{2}$. Johannes Zellner ${ }^{1} \cdot$ Felix Mayr $^{1}$ - Werner Krutsch ${ }^{1}$. \\ Leonard Achenbach $^{1} \cdot$ Rebecca WoehI $^{1} \cdot$ Michael Nerlich $^{1} \cdot$ Peter Angele $^{1,2}$
}

Published online: 20 February 2018

(c) Springer-Verlag GmbH Germany, part of Springer Nature 2018

\section{Correction to: Archives of Orthopaedic and Trauma Surgery https://doi.org/10.1007/s00402-018-2885-1}

With regards to Berardo Di Matteo, second author. The author's name is incorrectly listed on Pub-Med. The first and last name have been mixed up.
The original article can be found online at https://doi.org/10.1007/ s00402-018-2885-1.

Peter Angele

peter.angele@ukr.de

Matthias Koch

matthias.koch@ukr.de

Berardo Di Matteo

berardo.dimatteo@gmail.com

Jürgen Eichhorn

eichhorn@ogp.de

Johannes Zellner

johannes.zellner@ukr.de

Felix Mayr

felix.mayr@stud.uni-regensburg.de

Werner Krutsch

werner.krutsch@ukr.de
Correct first name is: B (on PubMed: BD.).

Correct last name is: Di Matteo (on PubMed: Matteo).

On SpringerLink the name is listed correctly, but on PubMed he is listed as Matteo BD.
Leonard Achenbach

leonard.achenbach@ukr.de

Rebecca Woehl

rebecca.woehl@ukr.de

Michael Nerlich

michael.nerlich@ukr.de

1 Department of Trauma Surgery, University Medical Centre Regensburg, Franz Josef Strauss Allee 11, 93042 Regensburg, Germany

2 Sporthopaedicum Straubing, Regensburg, Hildegard von Bingen Strasse 1, 93053 Regensburg, Germany

3 Center for Biological and Functional Reconstruction of the Knee, Humanitas Research and University Hospital, Rozzano, Italy 